\title{
Miranda
}

Revue pluridisciplinaire du monde anglophone /

Multidisciplinary peer-reviewed journal on the English-

speaking world

17 | 2018

Paysages et héritages de David Bowie

\section{Adam Barkman et Antonio Sanna (eds), A Critical Companion to Tim Burton}

\section{Gilles Menegaldo}

URL : http://journals.openedition.org/miranda/14507

DOI : $10.4000 /$ miranda. 14507

ISSN : 2108-6559

\section{Éditeur}

Université Toulouse - Jean Jaurès

\section{Référence électronique}

Gilles Menegaldo, «Adam Barkman et Antonio Sanna (eds), A Critical Companion to Tim Burton»,

Miranda [En ligne], 17 | 2018, mis en ligne le 02 octobre 2018, consulté le 16 février 2021. URL : http:// journals.openedition.org/miranda/14507 ; DOI : https://doi.org/10.4000/miranda.14507

Ce document a été généré automatiquement le 16 février 2021.

\section{(c) (i) () $\Theta$}

Miranda is licensed under a Creative Commons Attribution-NonCommercial-NoDerivatives 4.0 International License. 


\title{
Adam Barkman et Antonio Sanna (eds), A Critical Companion to Tim Burton
}

\author{
Gilles Menegaldo
}

\section{RÉFÉRENCE}

Adam Barkman et Antonio Sanna, (eds), A Critical Companion to Tim Burton. Lexington

Books, Lanham, Boulder, New York, London, 2017, 249 pages, ISBN: 978-1498552721.

1 Cet ouvrage collectif se présente sous la forme d'un volume relié de 249 pages. Il comporte un index, mais pas de bibliographie critique synthétique ni de filmographie. Les références bibliographiques se situent dans l'introduction et à la fin des différents chapitres. Les 21 contributeurs (certains articles sont co-écrits) sont pour la plupart des universitaires de différentes nationalités (dont deux chercheurs français).

2 L'ouvrage propose des contributions bien documentées et une pluralité d'approches critiques, ce qui ne nuit pas à la cohérence de l'ensemble. Les chapitres, de longueur parfois inégale, donnent une bonne idée de la spécificité tant thématique qu'esthétique du cinéma de Burton. Ils mettent l'accent sur certains aspects saillants de son univers filmique, en particulier le motif de la métamorphose du corps, ses diverses hybridations (homme/animal, homme/machine etc.), le motif de la création souvent couplé avec la monstruosité physique et/ou morale, la quête identitaire, le statut des personnages féminins. Les auteurs soulignent aussi l'influence de l'expressionnisme, la forte présence des tropes du gothique, le rôle du grotesque et mettent en exergue l'hybridité générique et la densité intertextuelle (le conte de fées, les films de série B, les films d'horreur du studio Hammer etc.).

3 L'introduction assez conséquente (16 pages) et dense, propose d'abord un bref état des lieux de la critique et présente ensuite l'œuvre de Burton film après film, par ordre chronologique. Le reste de l'introduction est consacré à une rapide description des 
différents chapitres. L'ouvrage comporte 20 chapitres structurés en trois sections: "Constructing Worlds» (6 chapitres), «Fairy Worlds and Nightmares " ( 7 chapitres), «Identity and the World» (7 chapitres).

4 La première section comporte des chapitres synthétiques centrés sur une question précise. Elle s'ouvre sur l'article de Jeffrey Andrew Weinstock qui rappelant la dualité des mondes chez Burton (le monde réel, celui de la surface, et le monde d'en dessous ou de l'au-delà, le «fantasy world» qui en est l'envers), met en lumière les caractéristiques spatiales de ces deux mondes, les contrastes architecturaux, mais aussi les différences de statut ontologique. L'auteur insiste sur la dimension verticale et en particulier les motifs de l'élévation et de la chute qui participent de la quête identitaire des personnages (Batman, Catwoman, Alice, Victor dans Corpse Bride, etc.).

Dans "The Abject, Carnivalesque, and Uncanny », Fran Pheasant-Kelly examine en particulier les représentations spatiales de la vie après la mort. Elle examine certains films de Burton au prisme des différents concepts évoqués dans le titre. Sleepy Hollow est lié au concept Kristévien d'abjection, Beetlejuice et Miss Peregrine étant plutôt associés au concept freudien d'inquiétante étrangeté (uncanny) tandis que Corpse Bride décline divers motifs carnavalesques. Selon l'auteur, Burton utilise ces modes pour figurer des formes alternatives de vie après la mort, des lieux de résurrection et de résistance. Les films étudiés associent une esthétique expressionniste et la « wound culture » (Mark Seltzer), la fascination pour le corps fragmenté, mutilé, la blessure et le trauma.

6 Dans « The Dark and the Darker: The Meaning and Significance of Dark and Light Colors in Tim Burton's Films", Orsolya Karacsony analyse l'emploi des couleurs, distinguant entre couleurs sombres et couleurs lumineuses, dichotomie qui selon elle reflète les traits distinctifs de chaque personnage, souvent un outsider confronté à un environnement différent et aliénant. Ainsi Edward, créature gothique au visage blafard et vêtu de noir est confronté à un monde suburbain aux couleurs pastel. Sabine Planka examine les références au surréalisme dans l'œuvre et relève plusieurs stratégies héritées de ce mouvement comme le choc inattendu entre deux situations ou les procédés du collage et du frottage. Le brouillage des frontières entre rêve et réalité constitue un autre trait commun tout comme la nature hybride des objets et des personnages. L'auteur compare certains motifs burtoniens avec divers tableaux de Magritte, Max Ernst ou encore Hans Bellmer.

7 Avec « Tim Burton's Artists of Death ", Elsa Colombani propose une étude stimulante sur le motif de la création artistique, se focalisant sur l'artiste criminel et destructeur à l'instar du Joker dans Batman ou de Benjamin Barker dans Sweeney Todd. Ainsi le Joker comme le cavalier sans tête se définissent par trois éléments : l'emploi d'un instrument tranchant, le désir de la défiguration et l'accumulation de cadavres. Le parallèle établi entre Edward et Sweeney Todd est tout aussi éclairant. La première section se clôt sur le chapitre de Andrew S. Powell centré sur la collaboration entre Tim Burton et Danny Elfmann. L'auteur examine en particulier l'interaction entre la musique diégétique (Prince dans Batman par exemple) et la musique non diégétique d'Elfmann. Il examine aussi certains procédés musicaux, les mélodies, l'harmonie et le rythme et met en lumière l'emploi d'instruments comme le célesta ou le thérémine.

8 La section 2 s'ouvre sur une belle analyse de l'univers cauchemardesque de Alice in Wonderland et Planet of the Apes. Antonio Sanna se livre d'abord à une présentation du diptyque Carrollien et de certaines versions filmiques, mettant aussi en relief la 
présence du motif darwinien de la lutte pour la vie et de l'adaptation au milieu, "survival of the fittest ». Il montre ensuite comment Burton exploite ce motif avec la résilience d'Alice confrontée à un monde cauchemardesque et violent. Le même concept darwinien est décliné dans Planet of the Apes où l'astronaute Léo Davidson, " héros réticent » est confronté à un monde de tyrannie et d'injustice qui inverse les relations traditionnelles de domination entre homme et singe. Christopher M. Cuthill examine les possibles résonances théologiques dans The Nightmare Before Christmas, au prisme de l'œuvre de Paul Tillich, en particulier de son expérience existentielle de la foi. L'auteur examine le parcours du protagoniste Jack Skellington, son vain désir de transformer Christmas Town et son retour à Halloween Town après une prise de conscience que l'auteur associe à une reconfiguration spirituelle.

9 Alissa Burger prolonge l'analyse du même film et se focalise sur la relation avec le conte de fées, établissant aussi un parallèle avec Corpse Bride. Elle montre que ces deux films subvertissent les codes du genre en confrontant les protagonistes à un monde autre reposant sur des valeurs inversées. L'accent est mis en particulier sur les personnages féminins (Sally, Victoria, Emily) qui mettent en crise les rapports de genre traditionnels et défient le pouvoir patriarcal. Nicole Pramik choisit comme objet Mars Attacks défini comme « fractured fairy tale ». Elle étudie ce film en relation avec Tolkien et ses trois principes structurant le conte merveilleux : "recovery", " escape", "consolation ». Selon l'auteur, le film évolue, passant du statut de simple comédie SF un peu "noire » à celui de conte héroïque classique comportant une dimension de satire sociale. Carl Sobocinski dans "The Heroic Journey of Ed Wood » définit d'abord le genre «biopic » avant de s'attacher au film de Burton qui rend hommage à un cinéaste marginal et excentrique à bien des égards. L'accent est mis sur le caractère absurde, voire «pataphysique» du film, mais aussi sur la dimension mythologique illustrée par la figure de Bela Lugosi ou encore celle du génie tutélaire incarné dans le film par Orson Welles. Ed Wood lui-même, confronté à de multiples épreuves est associé à une figure héroïque. L'auteur compare aussi le portrait établi par Burton avec la biographie du cinéaste, notant les différences et les zones d'ombre. Maria Dicieanu s'intéresse à la figure du héros déficient. Établissant un parallèle avec Prométhée, elle étudie les personnages " paranormaux» dans des œuvres moins balisées comme The Melancholic Death of Oyster Boy ou la série Stainboy, inspirée des dessins de Tim Burton, mais aussi dans des films plus récents comme Miss Peregrine. Ces personnages désireux de normalité sont cependant marqués par leur condition tragique jusqu'à la fin. La section se clôt sur l'article de Trip McCrossin consacré à ce dernier opus burtonien. L'auteur propose une analyse comparative du roman source de Ransom Riggs et de son adaptation, mais aussi une lecture du film au prisme du célèbre texte de Hannah Arendt, Eichman in Jerusalem: A Report on the Banality of Evil. L'auteur explore ainsi la relation au contexte historique de la Seconde Guerre mondiale et en particulier la persécution des juifs.

10 La troisième section s'ouvre sur le texte de Florent Christol consacré au premier long métrage de Burton, Pee-Wee's Big Adventure. L'auteur montre que, en dépit de l'apparente posture subversive du film qui propose une vision satirique de la petite ville américaine et aussi une vision critique et parodique des genres hollywoodiens, le discours sous-jacent est marqué par une idéologie conservatrice, impérialiste, imprégnée de stéréotypes. L'auteur se livre à une étude minutieuse de la fonction symbolique de différents objets fétichisés (comme le Gremlin jouet ou le portrait de George Washington près d'une statuette de pygmée) qui trahissent une vision passéiste 
et nostalgique d'un ordre révolu. Le parcours de Pee-Wee en quête de sa bicyclette, autre fétiche, trahit également un désir de retour à un ordre antérieur. Le film renforcerait aussi les stéréotypes raciaux et sociaux au lieu de les dénoncer. Le chapitre suivant est consacré au personnage excentrique de Willie Wonka que Radoslaw Osinski définit à l'aune de la figure du dandy. Le personnage mis en scène par Burton est un artiste plus soucieux d'esthétique que d'éthique. Il transforme l'espace de la fabrique en scène de spectacle où il officie en tant que maître de cérémonie. Wonka incarne ainsi un "dandy contemporain " selon Susan Sontag ("Notes on Camp ») qui célèbre ironiquement la contre-culture et dénonce, par sa posture théâtrale et ses excès, l'artificialité des normes sociales. Dans le chapitre suivant, Kyle Alkema et Adam Barkman se focalisent sur la figure de Batman mise en scène dans Batman et Batman Returns. Ils s'interrogent sur l'identité du super-héros et l'apport de Burton qui met l'accent sur la complexité psychologique du personnage incarné par Michael Keaton et suggère aussi que Bruce Wayne est le masque de Batman, non l'inverse. Les auteurs montrent aussi en quoi d'une part, Burton s'écarte du personnage créé par Bob Kane et comment, d'autre part Batman se construit en miroir avec les « super-villains » comme Penguin et surtout le Joker, antithèse absolue du héros masqué. Le personnage de Catwoman permet d'introduire la relation entre identité et liberté, mais aussi de souligner l'ambivalence des sentiments entre les deux personnages doubles, entre désir et détestation.

11 Siobhan Lyons (chapitre 17) revient sur deux films souvent évoqués dans l'ouvrage, The Nightmare Before Christmas et Edward Scissorhands et se propose d'analyser la figure de l'outcast à la lumière de la philosophie de Nietzsche, en particulier du concept de surhomme, et de celle de Heidegger qui développe un autre concept, celui de das Man (l'homme ordinaire). Ainsi, selon l'auteur, les héros burtoniens comme Edward ou Jack sont des figures individualistes subversives, des artistes créateurs s'opposant au conformisme d'une société qui rejette in fine leur singularité. Renée Middlemost met en évidence l'importance de la nostalgie et du sentiment de perte dans Beetlejuice et Edward Scissorhands. Le motif du «monstre sympathique » favorise l'identification du spectateur. Edward et Bételgeuse perturbent la routine quotidienne et mettent en évidence la vraie monstruosité, celle du conformisme de l'ordre domestique. Edward, en particulier, est l'objet de désirs transgressifs. Brent Peters et Adam Barkman examinent dans 5 films de Burton (Ed Wood, Sweeney Todd, Dark Shadows, Frankenweenie et Big Eyes) l'importance du déterminisme dans la trajectoire de protagonistes définis par leur démesure et leur caractère monomaniaque. Les auteurs tentent de démontrer que ces personnages qu'ils soient issus de la surnature ou pas, demeurent prisonniers de leurs obsessions et inconscients des conséquences (souvent catastrophiques) de leurs actes. En ce sens ils ne sont pas libres. Enfin Donna Mitchell examine la nature complexe et ambiguë des personnages féminins dans deux films d'animation en stopmotion (image par image), The Nightmare before Christmas et Corpse Bride. La métaphore de la « gothic doll » (poupée gothique) en tant que double est exploitée pour explorer la nature fragmentée et l'identité instable de personnages comme Sally qui se libère de son créateur, devient " an icon of self-transformation » et enfin le double et l'égal de Jack. De la même manière dans Corpse Bride, Victoria s'émancipe de l'autorité parentale alors qu'Emily, son double dans le monde des morts, choisit de se sacrifier et de revenir dans le cycle naturel, se transformant en une myriade de papillons.

Cet ouvrage propose une grande variété d'approches, souvent complémentaires. Les auteurs mobilisent de nombreuses sources théoriques et critiques et exploitent les 
travaux antérieurs sur Tim Burton, citant également les propos du cinéaste. Certains textes adoptent une approche philosophique ou même théologique, d'autres examinent l'idéologie ou la dimension de satire sociale, d'autres se concentrent sur des aspects formels (les couleurs, la musique). Plusieurs auteurs convoquent les gender studies ou encore la psychanalyse. Les différents chapitres sont de bonne tenue, bien structurés et argumentés et bien écrits. Certains sont moins convaincants, en particulier l'approche théologique.

On observe certaines lacunes. Rien n'est dit des tout premiers courts-métrages d'animation réalisés chez Disney et il est très peu question des deux courts métrages les plus connus, Vincent et Frankenweenie. En revanche, certains films (The Nighmare Before Christmas, Edward Scissorhands) sont largement plus étudiés que d'autres, d'où un déséquilibre et des répétitions ponctuelles. Les micro-analyses formelles sont relativement rares et limitées à quelques articles, notamment ceux qui concernent l'étude de l'espace. On peut aussi regretter l'absence totale d'illustrations dans un ouvrage consacré à un cinéaste célébré pour son pouvoir d'évocation visuelle.

En dépit de ces quelques réserves, nous recommandons fortement la lecture de ce livre qui offre des éclairages variés sur les films et constitue une contribution importante et novatrice à la connaissance de l'œuvre de Tim Burton.

\section{INDEX}

Mots-clés : cinéma, expressionnisme, surréalisme, gothique, conte de fée, animation, hybridité générique, intertextualité, fantastique, grotesque, macabre, carnavalesque, déterminisme, philosophie, théologie, mythologie, contre-culture, nostalgie

Keywords : cinema, expressionism, surrealism, gothic, fairy tale, animation, generic hybridity, intertextuality, fantastic, grotesque, macabre, carnivalesque, determinism, philosophy, theology, mythology, counter-culture, nostalgia

\section{AUTEURS}

\section{GILLES MENEGALDO}

Professeur émérite Université de Poitiers gilles@menegaldo.net 